\title{
The threshold-versus-duration curve is nonmonotonic
}

\author{
THOMAS R. CORWIN \\ New England College of Optometry, Boston, Massachusetts
}

\begin{abstract}
Detection thesholds for luminance pulses of either 50 or $250 \mathrm{msec}$ were measured, using a 2AFC procedure, in 20 subjects. For almost all subjects, 50 -msec pulses had lower thresholds than 250-msec pulses, demonstrating that the threshold-versus-duration curve has an undershoot.
\end{abstract}

Does the threshold-versus-duration curve decrease monotonically, or does it "undershoot," such that some intermediate-duration flash has the lowest threshold? This question has theoretical interest because of its implication about the form of the temporal impulse response function (e.g., Kelly \& Savoie, 1978).

According to the widely cited data of Graham and Kemp (1938) and Barlow (1958), the detection threshold for flashes decreases monotonically with duration and then levels off. More recent studies, however, report a small threshold undershoot for flashes of about $50 \mathrm{msec}$ (Kelly \& Savoie, 1978; Krauskopf, 1980; Rashbass, 1970). The data reported in these studies came from only four subjects. Because of this limited sample, and because the undershoot effect is small, its statistical reliability remains unclear. Furthermore, it is possible that some subjects show the effect and others do not. In a population of 80 subjects, Bowen and Markell (1980) found reliable nonmonotonic brightnessversus-duration functions in only $57 \%$ of those tested. In other subjects, brightness judgments varied with experimental conditions or were consistently monotonic. It is reasonable to assume that similar individual differences may exist in threshold-versus-duration curves.

\section{METHOD}

To test this possibility, we measured flash-detection thresholds in 20 subjects, under carefully controlled conditions. A large uniform field of moderately high photopic intensity was chosen in order to maximize temporal sensitivity. To produce flashes, a highly linear driver circuit (Beaty \& Corwin, 1983) supplied current pulses to a single green light-emitting diode (LED). The subjects viewed the LED centrally through a magnifying eyepiece, which produced a uniform circular field $15 \mathrm{deg}$ in diameter. The field's luminance was $57 \mathrm{~cd} / \mathrm{m}^{2}$, and the background was dark. Viewing was monocular with a natural pupil. Flashes were produced by incrementing the luminance of the entire field for a fixed duration, so that subjects had to rely entirely on time changes in luminance detection. Preliminary mea-

This research was supported in part by Grant EY 02366 and CVS Center Support Grant EY 01319. The author's mailing address is: New England College of Optometry, 424 Beacon Street, Boston, MA 02115. surements at a variety of flash durations had indicated that 50 -msec flashes had lower thresholds than flashes of longer duration. In the main study, therefore, $50-\mathrm{msec}$ flashes were presented in conjunction with a 250-msec long-duration comparison. The two durations were interleaved randomly in a session. Initial luminance levels for the two flash durations were set at approximately twice their eventual average thresholds. Consequently, most observers were unaware that two flash durations were being presented.

A two-interval forced-choice procedure was used, signaled by two 1-sec tones. Stimulus luminance was varied according to a Wetherill tracking procedure (Corwin, Kintz, \& Beaty, 1979). Luminances were increased or decreased in 5\% steps until eight reversals had taken place for each duration. The means of each peak-valley pair were used to calculate $t$ scores for threshold differences $(\mathrm{df}=6)$.

The subjects were recruited from staff and personnel of the Center for Visual Science at the University of Rochester. Each person was tested in a single session lasting 5-7 min, requiring an average of 80 trials.

\section{RESULTS AND DISCUSSION}

Despite considerable differences among individual thresholds, 19 of the 20 subjects were more sensitive to the 50 -msec flash than to the 250 -msec flash. The mean value of the threshold difference for all subjects was 0.17 $\log$ units.

Figure 1 is a scatterplot of the data. Open symbols denote statistically significant threshold differences ( $p<.05$, t test, two-tailed). Among the five subjects who did not show significant threshold differences, two had high thresholds for both durations (rightmost points in Figure 1). These subjects reported difficulty with fading of the stimulus field, perhaps due to retinal rivalry from a dominant contralateral eye. A third subject reported he was unable to hear the signal tones.

Overall, the data establish beyond reasonable doubt that, under our stimulus conditions, the thresholdversus-duration curve is reliably nonmonotonic for most observers. In a follow-up of their earlier study, Bowen, Sekuler, Owsley, and Markell (1981) discovered no group differences in flicker or motion sensitivity in subjects who had shown markedly different patterns of flash brightness estimates. They concluded that group differences in brightness estimates are due to differences 


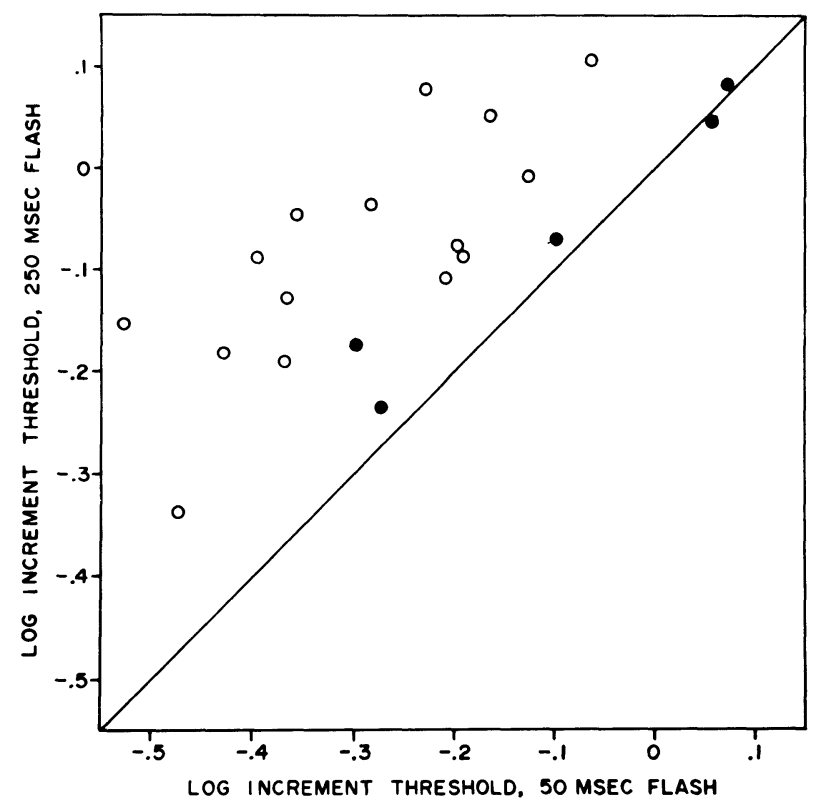

Figure 1. A scatterplot of 20 observers' detection thresholds for 50- and 250-msec flashes. Points lying above the diagonal represent cases in which the 50 -msec flash is more detectable than the 250 -msec flash. All points but one lie above the diagonal. For the open symbols, the threshold difference is statistically reliable at $\mathrm{p}<.05$ ( $\mathrm{t}$ test, two-tailed). For the filled symbols, p $>.05$. in criteria and not to differences in sensitivity for flashes of various durations. Our results directly confirm this conclusion.

\section{REFERENCES}

BARLOW, H. B. (1958). Temporal and spatial summation in human vision at different background intensities. Journal of Physiology, 141, 337-350.

Beaty, W. J., \& Corwin, T. R. (1983). An improved circuit for control of LED luminance. Behavior Research Methods \& Instrumentation, 15, 357-359.

Bowen, R. W., \& Markell, K. A. (1980). Temporal brightness enhancement studied with a large sample of observers. Perception \& Psychophysics, 27, 465-476.

Bowen, R. W., Sekuler, R., Owsley, C. J., \& Markell, K. A. (1981). Individual differences in brightness perception. Perception \& Psychophysics, 30, 587-593.

Corwin, T. R., Kintz, R. T., \& Beaty, W. J. (1979). Computeraided estimation of psychophysical thresholds by Wetherill tracking. Behavior Research Methods \& Instrumentation, 11, 526-528.

Graham, C. H., \& Kemp, E. H. (1983). Brightness discrimination as a function of the duration of the increment in intensity. Journal of General Physiology, 21, 635-650.

Kelly, D. H., \& Savoie, R. E. (1978). Theory of flicker and transient responses. Journal of the Optical Society of America, 68, 1481-1490.

KrAuSKOPF, J. (1980). Discrimination and detection of changes in luminance. Vision Research, 20, 671-677.

Rashbass, C. (1970). The visibility of transient changes of luminance. Journal of Physiology, 210, 165-186. 